\title{
Recognition and Treatment of Potentially Fatal Hyperkalemia in the Emergency Department
}

\author{
Waqas Jehangir ${ }^{\mathrm{a}, \mathrm{c}}$, Mohamed Osman ${ }^{\mathrm{a}}$, Dominique Jones ${ }^{\mathrm{b}}$, Mahmood Alam ${ }^{\mathrm{a}}$, Abdalla Yousif ${ }^{\mathrm{a}}$
}

\begin{abstract}
Acute hyperkalemia can be challenging to recognize clinically since its presentation may be vague. Hyperkalemia can be catastrophic in its outcome, leading to sudden death from cardiac arrhythmias if not recognized and treated emergently. This fact emphasizes the critical need to have a vigilant eye for these cases, provide emergent care, and have timely intervention in order to save patients at risk. We are presenting three cases with hyperkalemia with different outcomes.
\end{abstract}

Keywords: Hyperkalemia; Arrhythmia; Emergent; Hemodialysis

\section{Introduction}

Hyperkalemia is a common clinical problem, which is a result of impaired urinary potassium excretion due to acute or chronic kidney disease (CKD) and/or disorders or drugs that inhibit the renin-angiotensin-aldosterone axis [1]. Less commonly, redistributive hyperkalemia causes the movement of potassium out of the cells even though the total body potassium may be reduced. This is most commonly seen in uncontrolled hyperglycemia. Clinical manifestations of hyperkalemia vary from muscle weakness or flaccid paralysis [2], mimicking GuillainBarre syndrome, cardiac conduction abnormalities, and cardiac arrhythmias which can be fatal. These are mostly seen in patients with $\geq 7.0 \mathrm{mEq} / \mathrm{L}$ with acute or chronic kidney failure and other co-morbidities leading to sudden rise in serum potassium levels. Patients with skeletal muscle or cardiac manifestations typically have one or more of the characteristic electrocardiographic abnormalities associated with hyperkalemia.

Manuscript accepted for publication February 25, 2015

aRaritan Bay Medical Center, Perth Amboy, NJ 08861, USA

bRutgers University School of Related Professions, Newark, NJ, USA

${ }^{\mathrm{c} C}$ Corresponding Author: Waqas Jehangir, Raritan Bay Medical Center, Perth Amboy, NJ 08861, USA. Email: wjehangir@hotmail.com

doi: http://dx.doi.org/10.14740/jmc2078w

\section{Case Reports}

\author{
Case 1 (the good)
}

A 72-year-old female with past medical history of DVT, hypertension, and right-sided middle lobe lung cancer that had been treated with chemotherapy was admitted to the hospital for left knee pain secondary to osteomyelitis. She had exertional shortness of breath and was on home oxygen by nasal cannula. The patient was also on coumadin for deep venous thrombosis of the left calf. She had history of tobacco smoking in the past. On physical exam, the patient was afebrile with blood pressure 130/70 $\mathrm{mm} \mathrm{Hg}$, pulse 80/min, and respirations 18/min. There was severe tenderness noted over the medial tibial plateau of the left knee with minimal joint effusion and weakness on the lower extremity; range of movement was reduced. On her third day of admission, she started having chest pain and rapid response team was called. An electrocardiograph (ECG) was performed which showed ST elevation and a code heart was called. The ECG was reviewed by the cardiologist who suspected that it was hyperkalemia, not ischemic changes (Fig. 1). The patient was given intravenous insulin, sodium bicarbonate, and calcium gluconate. The patient was also given sodium polystyrene sulfonate and her potassium level came out to be $9.3 \mathrm{mmol} / \mathrm{L}$. The repeat serum potassium level with initial treatment came down to $6.4 \mathrm{mmol} / \mathrm{L}$ requiring additional treatment. The repeat ECG showed resolution of acute ST changes (Fig. 2). Later on the potassium was $4.7 \mathrm{mmol} / \mathrm{L}$. Patient had no adverse outcome with hyperkalemia.

\section{Case 2 (the bad)}

This is a 65-year-old male with past medical history significant for ischemic CVA, hyperlipidemia, type 2 diabetes mellitus, end-stage renal disease, depression, seizure disorder and hypertension. He was admitted to the emergency room after he was noted to have seizure. This lasted, according to the daughter, for 1 - 2 min and she characterized it as a grand mal seizure. The vital signs showed the blood pressure 180/64 mm $\mathrm{Hg}$, pulse rate $58 / \mathrm{min}$, respiratory rate $16 \mathrm{~min}$, and temperature 98.4. The physical exam was otherwise unremarkable. At the time of admission, laboratory data showed $\mathrm{Hb} 9 \mathrm{~g} / \mathrm{dL}$, Hct 


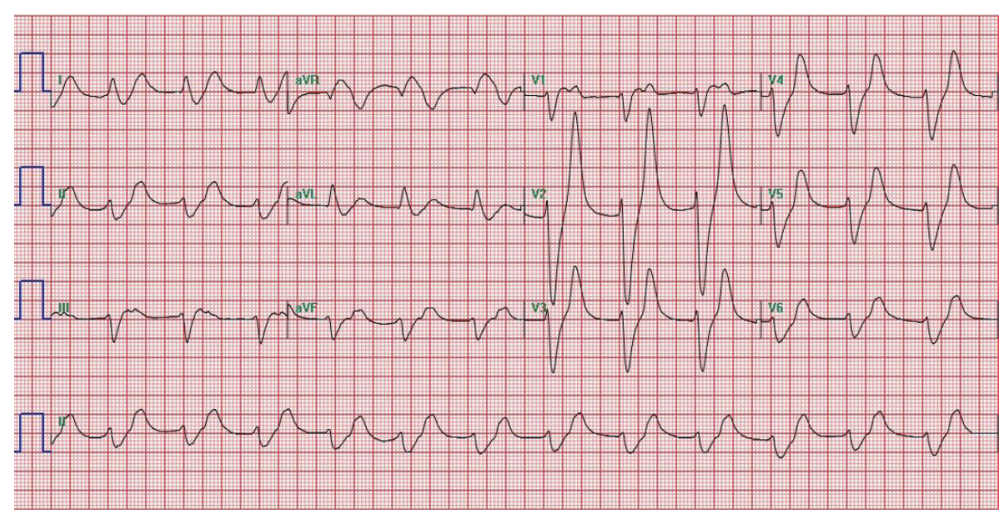

Figure 1. ECG showing tall tented T waves.

$27.1 \%$, WBC $4,000,000 / \mu \mathrm{L}$, platelets $136,000 / \mu \mathrm{L}$, serum creatinine 2.6 and serum potassium $3.9 \mathrm{mmol} / \mathrm{L}$. The patient was admitted for breakthrough seizure. During his hospital stay, the patient had a cardiopulmonary arrest and ACLS was conducted. The ECG showed sine wave pattern (Fig. 3), and the potassium was found to be $6.8 \mathrm{mmol} / \mathrm{L}$. Calcium gluconate, insulin and sodium bicarbonate were given and emergent hemodialysis was performed, which saved the patient's life. The repeat ECG (Fig. 4) showed complete resolution of the arrhythmia.

\section{Case 3 (the ugly)}

This is a 66-year-old male with a past medical history of hypertension, diabetes, and atrial fibrillation. He was on rivaroxiban, digoxin, amiodarone, and metropolol. He was recently displaced to a motel after his home flooded and became increasingly lethargic. EMS was called by companions at the motel and in the field the patient had a heart rate of 10 . He responded to IV atropine, and was placed on transcutaneous temporary pacemaker in the emergency department. The patient was immediately taken to catheterization lab for trans-venous pacemaker insertion, which did not result in significant hemodynamic improvement and patient remained hypotensive. He was started on vasopressors for hypotension and was placed on ventilator for agonal breathing. Shortly after, he suffered a cardiac arrest and rhythm showed sine waves shortly prior to the arrest. ACLS was provided that failed to restore spontaneous circulation. The potassium level was discovered to be $7.9 \mathrm{mmol} / \mathrm{L}$ from the sample drawn in the ER and rhythm strip is shown (Fig. 5).

\section{Discussion}

The above mentioned cases suggest that patients with lifethreatening hyperkalemia generally present with vague symptoms with numerous co-morbidities. This problem may further complicate the issue in those patients who present in the emergency department and their medical history is unknown. Patients presenting with hyperkalemia typically have nonspecific symptoms consisting of chest pain, fatigue, weakness, GI complaints and impaired cardiac or muscular function. The high index of suspicion and early diagnosis and treatment would result in better outcomes for hyperkalemia. Immediate hemodialysis is needed in hyperkalemic patients with life-threatening arrhythmias in order to save their life.

These patients had numerous medical illnesses. However, the renal failure of any etiology is the single most important disease that results in hyperkalemia in a susceptible patient. Hyperkalemia is frequently seen, especially in the emergency department, and is typically due to disturbances in potassium intake, potassium excretion and transcellular shifts [3]. Acute

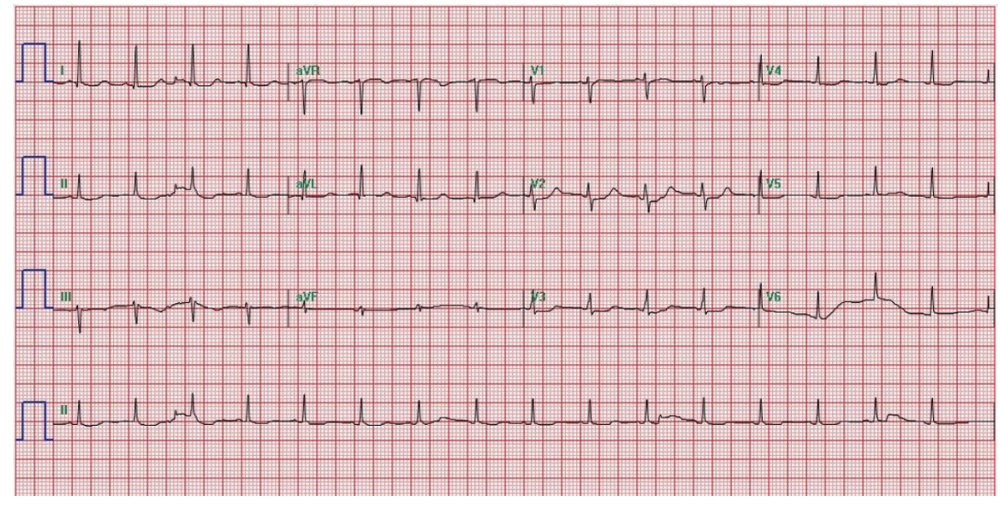

Figure 2. ECG showing resolution of tall tented T waves. 


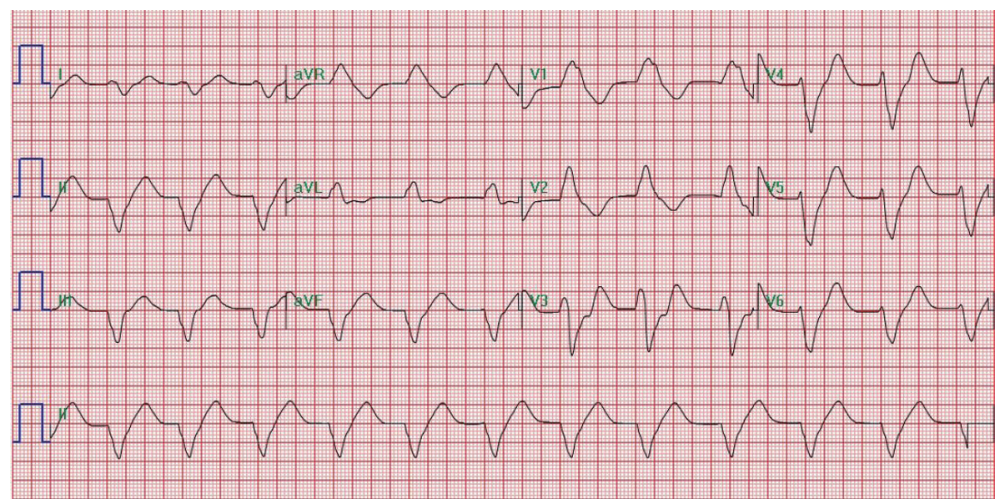

Figure 3. ECG showing sine wave pattern.

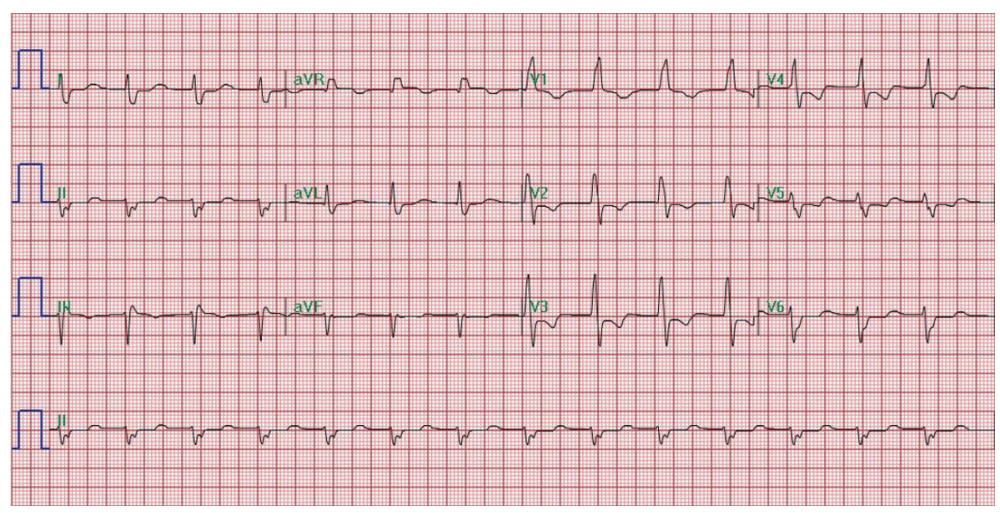

Figure 4. ECG showing complete resolution of sine wave pattern.

metabolic acidosis, drugs interfering with the excretion of potassium in already compromised renal function, and increased dietary intake of potassium can lead to the critical serum levels of potassium that leads to life-threatening arrhythmias. Insulin is a key hormone in promoting potassium uptake into cells but during insulin deficiency, in diabetes mellitus, there is a redistribution of potassium from intracellular to extracellular space leading to hyperkalemia [3]. The classic ECG changes in a patient with hyperkalemia is considered a good indication for emergent treatment $[3,4]$. Severe symptoms do not occur until serum potassium levels reach $7.0 \mathrm{mmol} / \mathrm{L}$ or above. There are many individual patient variables, and a rapid rate of rise is more dangerous than a slowly rising level [3]. The initial ECG findings are peaked T waves. As the potassium level increases, widened PR intervals, loss of P waves, ST segment changes and a widened QRS can be observed [3]. The widened QRS progresses to a "sine wave", which was seen in two of our patients, and ending in ventricular fibrillation and cardiac arrest when the levels reach $8.0-10.0 \mathrm{mmol} / \mathrm{L}$ [3].

It is seen that the urgent treatment for hyperkalemia is with intravenous glucose and insulin [3]. Two of our patients were given intravenous insulin, calcium gluconate and sodium bircarbonate whose ECG had suggested hyperkalemic changes. Initial treatment with above mentioned therapy for hyperkalemia may prevent progression of ECG changes; however, access serum potassium must be dealt with and its removal either by using gastrointestinal binding resin like polystyrene sulfonates or in many cases an emergent bedside hemodialysis may be required in saving the patients' lives. In ECG abnormalities, calcium is the mainstay of cardiac stabilization to restore resting membrane potential [4].

The urgency of treatment of patients with hyperkalemia does not depend on the serum potassium levels. In fact the ECG changes dictate the urgency of treatment. The classical sine wave presentation must be recognized from idioventricular rhythm that we invariably find in patients with complete

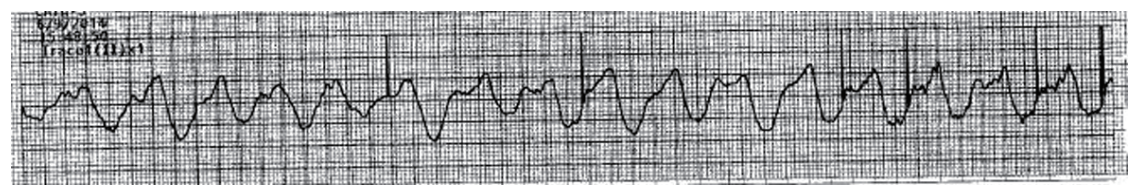

Figure 5. ECG showing widening of QRS complex and sine wave pattern. 
heart block since treatment will be totally different and cardiac pacing in critical hyperkalemia with QRS widening will not respond to electronic pacing [5]. Rather emergent hemodialysis will be lifesaving as we learnt from two of our three cases presented in this paper. An understanding of life-threatening hyperkalemia and early recognition of arrhythmia is the key to success in these patients. Appropriate treatment will lead to the prevention of morbidity and mortality [3].

\section{Conflict of Interest}

The authors declare that there is no conflict of interests regarding the publication of this article.

\section{References}

1. Kamel KS, Wei C. Controversial issues in the treatment of hyperkalaemia. Nephrol Dial Transplant. 2003;18(11):2215-2218.

2. Finch CA, Sawyer CG, Flynn JM. Clinical syndrome of potassium intoxication. Am J Med. 1946;1:337-352.

3. Schaefer TJ, Wolford RW. Disorders of potassium. Emerg Med Clin North Am. 2005;23(3):723-747, viii-ix.

4. Medford-Davis L, Rafique Z. Derangements of potassium. Emerg Med Clin North Am. 2014;32(2):329-347.

5. Ahee P, Crowe AV. The management of hyperkalaemia in the emergency department. J Accid Emerg Med. 2000;17(3):188-191. 